\title{
Super-Rough Dynamics on Tumor Growth
}

\author{
Antonio Brú, ${ }^{1, *}$ Juan Manuel Pastor, ${ }^{2}$ Isabel Fernaud, ${ }^{3}$ Isabel Brú, ${ }^{4}$ Sonia Melle, ${ }^{2}$ and Carolina Berenguer ${ }^{5}$ \\ ${ }^{1}$ CIEMAT, Avenida Complutense, 22, 28040 Madrid, Spain \\ ${ }^{2}$ Departamento de Física Fundamental, UNED, c/Senda del Rey s/n, 28040 Madrid, Spain \\ ${ }^{3}$ Instituto Ramón y Cajal, CSIC, Madrid, Spain \\ ${ }^{4}$ Hospital SS Ntra. del Prado, Talavera de la Reina, 45600, Spain \\ ${ }^{5}$ Facultad de Ciencias, Universidad de Alicante, 03690 Alicante, Spain
}

(Received 22 May 1998)

\begin{abstract}
The growth of a cultivated typical brain tumor is studied in this work. The tumor is analyzed both dynamically and morphologically. We have measured its fractal dimension to be $d_{f}=1.21 \pm 0.05$. From its dynamical behavior we determine the scaling critical exponents of this circular symmetry system which are compatible with the linear molecular beam epitaxy universality class. A very important feature of tumor profiles is that they are super-rough, which constitutes the first $(1+1)$ dimensional experiment in literature with super-roughness. The results obtained from the dynamics study make manifest two very surprising features of tumor growth: Its dynamics is mainly due to contour cells and the tendency of an interface cell to duplicate is a function of the local curvature. [S0031-9007(98)07545-0]
\end{abstract}

PACS numbers: 87.22.As, 47.53.+n, 47.54.+r, 68.35.Ct

Over the past two decades, many systems exhibiting rough interfaces in their development process have been successfully described by means of scaling analysis, a powerful tool used in the study of fractal geometry. In this way processes such as fluids invading porous media, fire fronts, crystal growth, etc. [1], have been understood. In the field of biology, we can find a large variety of systems which develop rough interfaces. Among the most interesting and important ones are growing tumors. The shape of the tumor profile will allow us to study and classify the type of tumor according to its geometry and dynamics. The contour form is a valuable indication of the tumor dynamics behavior, something that has also been observed in tumoral cells. The form of the contour cell determines the number and type of cell exchange channels. Moreover, the morphology of single cells may determine its malignant nature, as we learned in a very enlightening paper of Losa et al. [2]. This result has been extrapolated to the case of tumors to predict their malignant nature. There are several works where the fractal dimension of tumors has been measured with the aim to classify them and determine their malignant nature [3]. On the other hand, there have been different attempts to construct a mathematical model describing tumor growth [4-9], but they are too restrictive in their hypothesis. In this paper we propose a purely descriptive mathematical model derived from the study of the time evolution of tumor growth, which will serve to draw some conclusions about the mechanisms of this growth process.

We have grown four brain tumors in vitro from the cellular stable line C6 of rat astrocyte glioma. Approximately $10^{3}-10^{4}$ partially dissociated cells were plated on $35 \mathrm{~mm}$ diameter Petri dishes, in a volume of $5 \mu \mathrm{l}$ of medium (a mixture of Dulbecco's modified Eagle's medium (DMEM) and F12 Ham's mixture (F12) in a
1:1 ratio) in a $5 \% \mathrm{CO}_{2}$ and $95 \%$ humidity atmosphere. Once attached, fresh medium was added up to a volume of $2 \mu \mathrm{l}$, and the cells were maintained in these conditions for several days. This procedure allowed cells to grow mainly on the plate surface; i.e., for our purposes the tumor can be considered a two-dimensional system. The four tumors were photographed during their growth by using an inverted microscope equipped with a contrast filter and a coupled photocamera. We will henceforth label these tumors $A, B, C$, and $D$. Growing times range from about 50 hours (tumor $A$ ) up to 311 hours (tumor $D$ ). The analysis of the tumor evolution is based on the shape of their profiles. The photographs were scanned into a personal computer, achieving a final resolution of $1.3 \mu \mathrm{m} / \mathrm{pixel}$, and the profiles were hand traced. Some typical tumor profiles are shown in the inset of Fig. 1.

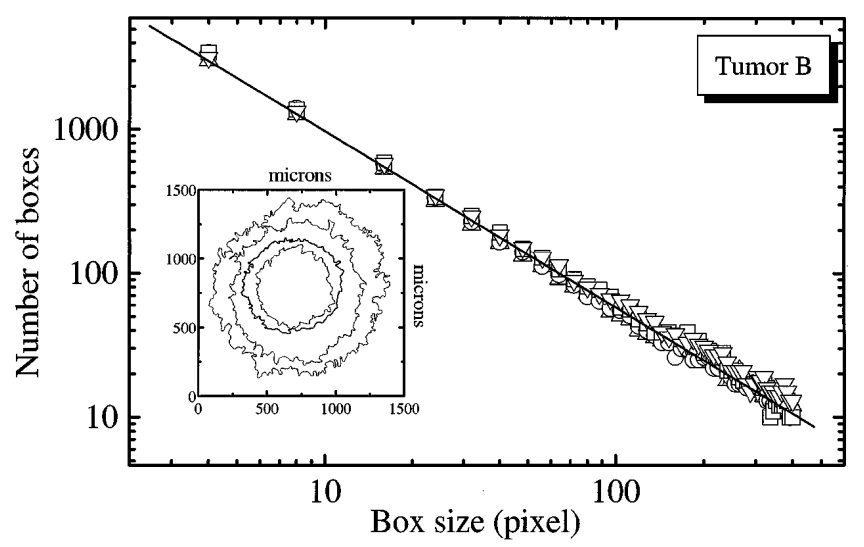

FIG. 1. Fractal dimension of the tumor $B$ calculated with the box-counting method at $t=0 h$ (up triangles), $21 h$ (circles), $29 h$ (squares), and $52 h$ (down triangles). The inset shows snapshots of tumor interface corresponding at the same times. 
In order to characterize the tumors morphometrically we have used the box-counting fractal dimension. In Fig. 1 we show the box counting analysis results for the interface of tumor $B$ at four different times. The value obtained for the fractal dimension is $d_{f}=1.21 \pm 0.05$. The same analysis has been made on the other three tumors, and the values obtained for $d_{f}$ are the same within the error bars. We stress that the measured $d_{f}$ value is independent of both time and experiment.

To describe the dynamics of tumor growth we study the scale-invariant behavior of the profiles. The dynamics of many physical systems exhibiting rough interfaces can be characterized by a set of critical exponents obtained from scale-invariant properties of certain physical quantities. The first one is the mean tumor radius, the first order moment of the interface position, $\langle r\rangle=N^{-1} \sum_{i=1}^{N} r_{i}(t)$, where $N$ is the number of points of the tumor interface and $r_{i}$ are the distances of these points from the center of mass of the tumor. Another important quantity is the second order moment of the interface position as a function of the arc length $l$ and time $t$ :

$$
w(l, t)=\left\{\frac{1}{l} \sum_{r_{i} \in l}\left[r_{i}(t)-\left\langle r_{i}\right\rangle_{l}\right]^{2}\right\}_{L}^{1 / 2},
$$

where $\langle\cdot\rangle_{l}$ means the local average of subsets of arc length $l$, and $\{\cdot\}_{L}$ is the average over all of the system. This quantity measures the interface width and provides a measure of local fluctuations of the interface at about its local average value. A system with circular symmetry showing negligible overhangs compared with the system size behaves like a linear system with a time dependent size. This result has been tested using different methods for determining the critical exponents [10]. The width function of these rough interfaces, depending on the arc length $l$ and time $t$, shows the scaling behavior

$$
w(l, t)= \begin{cases}t^{\beta} & \text { if } t \ll t_{s}, \\ l^{\alpha} & \text { if } t \gg t_{s} .\end{cases}
$$

with $\alpha$ the roughness exponent, $\beta$ the growth exponent, and $t_{s}$ the saturation time which depends on the window size, where $z$ is the dynamical exponent, which characterizes the time scaling behavior of the lateral correlation length, $l_{c} \sim t^{1 / z}$. These three critical exponents are related through $z=\alpha / \beta$, as in linear geometry. Although this description is valid for a great variety of physical systems, there exist some cases in which it is not valid. When the local width $w(l, t)$ differs from the global width $w(L, t)$, we can define $\alpha_{\text {loc }}$ and $\alpha$, the local and global roughness exponents, respectively, as

$$
w(l, t) \sim l^{\alpha_{\mathrm{loc}}}, \quad w(L, t) \sim L^{\alpha}, \quad t \gg t_{s},
$$

with $L$ being the whole contour length of the circular interface.

There are some systems in which the surface has a global roughness exponent $\alpha>1$ [1,11-13]. These systems are termed super-rough. In these cases, the local surface width does not saturate as in (3), but crosses over to a new behavior in the intermediate time regime $l^{z} \ll$ $t \ll L^{z}$, characterized by a different growth exponent $\beta_{*}, w\left(l, t \gg l^{z}\right) \sim l t^{\beta_{*}}$, where $\beta_{*}=\beta-\alpha_{l o c} / z$. A scaling showing this behavior is known as anomalous scaling [14]. The interface Fourier transform gives us the power spectrum

$$
S(k, t)=k^{-(2 \alpha+1)} s\left(k t^{1 / z}\right),
$$

where $s$ is the structure factor which shows the scaling behavior

$$
s(u)= \begin{cases}\text { const } & \text { if } u \gg 1, \\ u^{-(2 \alpha+1)} & \text { if } u \ll 1 .\end{cases}
$$

By applying this scaling analysis to the tumors we obtain some very interesting results. In Fig. 2 we can observe the time evolution of the mean radius, measured from the tumor center of mass. Note that the four curves have been shifted in order to match the initial radius at $t=t_{0}$. The radius grows linearly with time [15]. As shown in Fig. 2, the interface speed growth obtained is $\langle v\rangle=2.9 \pm 0.1 \mu \mathrm{m} / \mathrm{h}$. This result is striking because the growth rate is assumed to be exponential in tumor literature $[16,17]$.

In Fig. 3 we plot the local width of the interfaces of tumor $D$ at several times. We can establish for the local roughness exponent the value $\alpha_{\text {loc }}=0.87 \pm 0.05$. Notice that $d_{f}+\alpha_{\text {loc }}=2.08 \pm 0.10$, in good agreement with the exact result 2 (the Euclidean dimension) [18]. The global interface roughness exponent, $\alpha$, is obtained from the power spectrum of the tumor interfaces. As shown in Fig. 4 the power spectrum decays as $k^{-m}$ with $m=2 \alpha+1=4.0 \pm 0.2$, hence $\alpha=1.5 \pm 0.1$.

This result $(\alpha>1)$ indicates that tumor interfaces are super-rough, i.e., they exhibit anomalous scaling. Super-rough interfaces have been observed experimentally in crystal growth in $(2+1)$-dimensions, where super-roughness is marginal $[19,20]$.

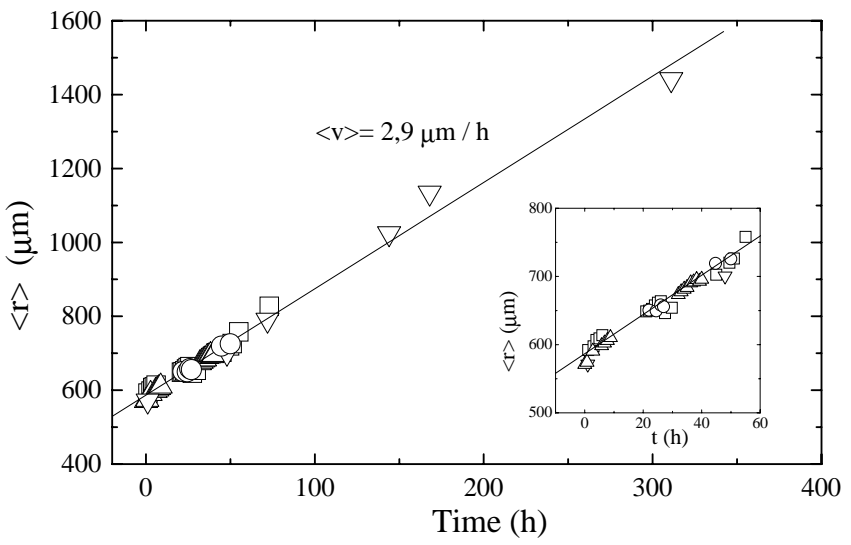

FIG. 2. Time evolution of the tumors mean radii showing a linear behavior [tumor $A$ (up triangles), tumor $B$ (circles), tumor $C$ (squares), and tumor $D$ (down triangles)]. In the inset we can see the fit for early times. 


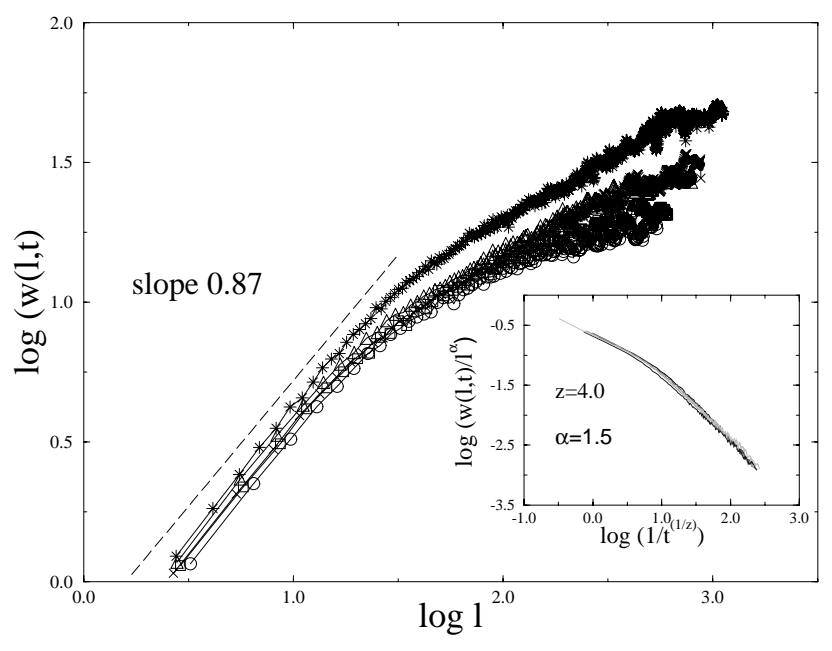

FIG. 3. Local width of tumor $D$ vs window size at times = $48 h$ (circles), $72 h$ (squares), 144h (triangles), 168h (pluses), and $311 h$ (stars). From the initial slope of the curves we obtain the local roughness exponent $\alpha_{\text {loc }}=0.87 \pm 0.05$. In the inset, data are collapsed using exponents $\alpha=1.5$ and $z=4.0$.

We can obtain $z$ from a scaled local width, $w(l, t) / l^{\alpha}$, vs scaled window size, $l / t^{1 / z}, \log$-log plot. According to (2) and (3), the local interface width curves at different times should collapse onto one curve which exhibits two different regimes, each with a characteristic decay. To measure the dynamical exponent, $z$, we consider tumor $D$ because of its larger growth time. The inset of Fig. 3 shows the collapse of tumor $D$, using $\alpha=1.5$ and $z=4.0$. The exponent of the first regime is $m=$ $\alpha_{\text {loc }}-\alpha=-0.55 \pm 0.10$ and that of the second regime is $m=-\alpha=-1.4 \pm 0.1$. In the same way, and to corroborate these latest values, we can obtain $\alpha$ and $z$

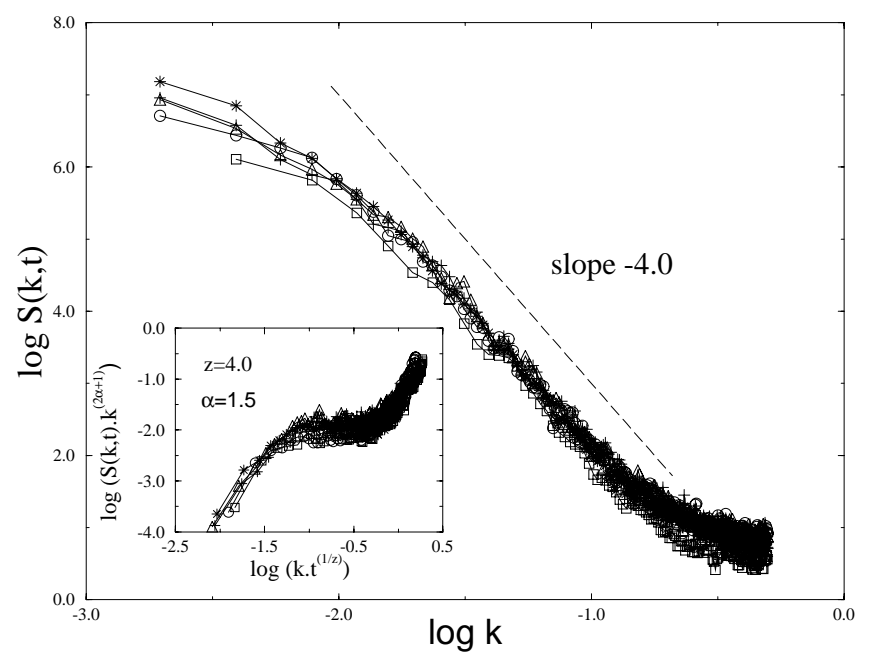

FIG. 4. Structure factor at times $=48 h$ (circles), $72 \mathrm{~h}$ (squares), 144h (triangles), 168h (pluses), and $311 h$ (stars) of tumor $D$. The shape of the curves provides us a global roughness exponent of $\alpha=1.5 \pm 0.1$. In the inset, data are collapsed using exponents $\alpha=1.5$ and $z=4.0$. from the collapse of the power spectrum of the interfaces. It can be seen that the spectrum curves at different times collapse onto a single universal curve. In the inset of Fig. 4 we show a log-log plot of $S(k, t) k^{2 \alpha+1}$ vs $k t^{1 / z}$ for $\alpha=1.5$ and $z=4.0$.

In summary, we have obtained the following set of critical exponents: $\alpha_{\text {loc }}=0.87 \pm 0.05, \alpha=1.5 \pm 0.1$, $z=4.0 \pm 0.2, \beta=0.375 \pm 0.03$, and $\beta^{*}=0.15 \pm$ 0.05 . This set of critical exponents values is compatible with the well-known linear MBE (molecular beam epitaxy) growth model, which describes growth processes dominated by surface diffusion and deposition, as in crystal growth. This universality class has been found in some deposition models $[11,21,22]$. The continuum equation which describes this universality class is

$$
\frac{\partial h}{\partial t}=-K \frac{\partial^{4} h}{\partial x^{4}}+F+\eta(x, t),
$$

where $h$ is the interface height, $K$ is the surface diffusion coefficient, and $\eta(x, t)$ is a random noise with $\langle\eta(x, t)\rangle=0$ and correlations $\left\langle\eta(x, t) \eta\left(x^{\prime}, t^{\prime}\right)\right\rangle=$ $2 D \delta\left(x-x^{\prime}\right) \delta\left(t-t^{\prime}\right)$. In our case $F$ represents a cell division rate. This linear equation can be exactly solved by using Fourier transformation, giving $\alpha=1.5$ and $z=4$, i.e., $\beta=3 / 8$. In atomic deposition described by MBE processes, atomic diffusion depends only on the number of bonds which must be broken for diffusion to take place instead of the local height of the interface. We can gain further knowledge of the microscopic growth process by means of the local curvature models [22,23], which considers nonequilibrium growth models driven by deposition and surface diffusion. In this model, the relaxation process follows the rule that any particle deposited at the interface will choose the site that increases the local curvature of the surface between itself and its nearest neighbors. The number of bonds a particle may form increases with the local curvature of the interface at that point. If the local curvature radius is positive the atom has a large number of neighbors and it is able to diffuse easily. In the case of tumors, the cell division plays the role of both deposition and surface diffusion. Depending on the local curvature, the interface cells will have, in some sense, a larger probability of duplicating. A high positive local curvature corresponds to a high cell duplication probability and vice versa. This result reveals a new surprising and important feature of tumor growth.

Finally, we have shown that this type of tumor has a dynamical behavior described by a mathematical model. On the other hand, it constitutes the first $(1+1)$-dimension experiment developing super-rough interfaces. All of these results can be effectively achieved only in cell culture, although it is important to assess the significance of the results for the growth behavior of cells in animals. The final goal of our research is to classify tumors according to their dynamical behavior, as well as to establish a connection between the physical environment of a tumor 
and the mathematical parameters. The latter would have outstanding medical consequences as it reveals the mechanism to control - or even to stop - the growth of a tumor.

We would like to thank Dr. M. A. Rodríguez, Dr. J. A. Cuesta, Dr. R. Cuerno and Dr. J. M. López for helpful discussions, and Dr. J. L. Jorcano, Carmen Segrelles, and Dr. Jesús Paramio for their help in the growth and treatment of the tumors.

*Electronic address: bruno@ciemat.es

[1] A.-L. Barabasi and H.E. Stanley, Fractal Concepts in Surface Growth (Cambridge University Press, Cambridge, England, 1995).

[2] G. A. Losa, G. Baumann, and Th. F. Nonnemacher, Pathol. Res. Pract. 188, 680 (1992).

[3] R. Sedivy, Wien. Klin. Wochenschr. 108, 547 (1996); P. Leskovar, D. K. Klaeßen, and J. Bielmeier, Z. Onkol. 28, 113 (1996); G. Landini and J. W. Rippin, J. Pathol. 179, 210 (1996).

[4] Z. Bajzer, M. Marusic, and S. Vuk-Pavlovic, Math. Comput. Model. 23, 31 (1996).

[5] H. M. Byrne, Math. Biosci. 144, 83 (1997).

[6] J. A. Adam, Math. Biosci. 86, 183 (1987); J. A. Adam, Math. Biosci. 130, 213 (1987).

[7] H. P. Greenspan, Stud. Appl. Math. 52, 317 (1972).

[8] J. Landry, J.P. Freyer, and A. M. Shuterland, Cell Tissue Kinetics 15, 585 (1982).

[9] D. L. S. McElwain and L. E. Morris, Math. Biosci. 39, 147 (1978).

[10] J. M. Pastor and A. Brú (to be published).
[11] S. Das Sarma, S. V. Ghaisas, and J. M. Kim, Phys. Rev. E 49, 122 (1994).

[12] J. M. López, Phys. Rev. E 52, 1296 (1995).

[13] J. Buceta, J. M. Pastor, M. A. Rubio, and F. J. de la Rubia, Physica (Amsterdam) 113D, 166 (1998).

[14] J. M. López, M. A. Rodríguez, and R. Cuerno, Physica (Amsterdam) 246A, 329 (1997).

[15] A. Brú, I. Fernaud, J. Pastor, I. Brú, C. Berenguer, and M.J. Fernaud, in XXVI Reunión Bienal de la Real Sociedad Española de Física (Ed. Universidad de Las Palmas, Las Palmas de Gran Canaria, 1997).

[16] R. I. Freshney, Animal Cell Culture (IRL Press, Oxford, 1986); W. B. Jakoby and I.H. Pastan, Cell Culture (Academic, New York, 1979).

[17] The linear growth can be explained with a simple argument. The number of cells in a tumor is $\pi\left(R / r_{0}\right)^{2}$, where $R$ is the tumor radius and $r_{0}$ the cell "radius," when $R \gg r_{0}$. If growing take place at the border the growing rate will be proportional to the number of perimeter tumor cells $2 \pi R / r_{0}$. Hence, $d\left[\pi\left(R / r_{0}\right)^{2}\right] / d t=K 2 \pi R / r_{0}$, i.e., $d R / d t=K r_{0}$. Therefore $R=R(t=0)+K r_{0} t$.

[18] F. Family and T. Viseck, Dynamics of Fractal Surfaces (World Scientific, Singapore, 1991).

[19] H.-N. Yang, Y.-P. Zhao, G.-C. Wang, and T.-M. Lu, Phys. Rev. Lett. 76, 3774 (1996).

[20] J. H. Jeffries, J.-K. Zuo, and M. M. Craig, Phys. Rev. Lett. 76, 4931 (1996).

[21] J. G. Amar, P.-M. Lam, and F. Family, Phys. Rev. E 47, 3242 (1993).

[22] E. Moro, R. Cuerno, and A. Sánchez, Phys. Rev. Lett. 78, 4982 (1997).

[23] J. M. Kim and S. Das Sarma, Phys. Rev. Lett. 72, 2903 (1994). 